\title{
Correction to: Selective ethanol gas sensing performance of flower-shaped CuO composed of thin nanoplates
}

\author{
Ahmad Umar ${ }^{1,2, *}$, A. A. Ibrahim ${ }^{1,2}$, Hassan Algadi ${ }^{2,3}$, Umesh T. Nakate ${ }^{4}$, Sandip P. Choudhury ${ }^{5}$, \\ Turki Alsuwian ${ }^{3}$, Hasan Albargi, ${ }^{2,6}$, Mabkhoot A. Alsaiari, ${ }^{2,7}$, and S. Baskoutas ${ }^{8}$ \\ ${ }^{1}$ Department of Chemistry, Faculty of Science and Arts, Najran University, P.O. Box 1988, Najran 11001, Kingdom of Saudi Arabia \\ ${ }^{2}$ Promising Centre for Sensors and Electronic Devices (PCSED), Najran University, P.O. Box 1988, Najran 11001, Kingdom of Saudi \\ Arabia \\ ${ }^{3}$ Department of Electrical Engineering, Faculty of Engineering, Najran University, Najran 11001, Kingdom of Saudi Arabia \\ ${ }^{4}$ Solar Energy Research Center, School of Semiconductor and Chemical Engineering, Jeoonbuk National University, 567 \\ Baekjedaero, Deokjin-gu, Jeonju-si, Jeollabuk-do 54896, Republic of Korea \\ ${ }^{5}$ National Institute of Technology Nagaland, Dimapur, India \\ ${ }^{6}$ Department of Physics, Faculty of Science and Arts, Najran University, P.O. Box 1988, Najran 11001, Kingdom of Saudi Arabia \\ ${ }^{7}$ Empty Quarter Research Unit, Department of Chemistry, College of Science and Arts in Sharurah, Najran University, Sharurah, \\ Kingdom of Saudi Arabia \\ ${ }^{8}$ Department of Materials Science, University of Patras, Rio, 26504 Patras, Greece
}

Published online:

3 July 2021

(C) Springer Science+Business

Media, LLC, part of Springer

Nature 2021

\section{Correction to:}

\section{J Mater Sci: Mater Electron}

https://doi.org/10.1007/s10854-021-06249-y
Publisher's Note Springer Nature remains neutral with regard to jurisdictional claims in published maps and institutional affiliations.

The affiliations of two authors, Dr. Hassan Algadi and Dr. Turki Alsuwian, are missing from the published article. The correct affiliations for the authors are provided in this erratum.

The original article can be found online at https://doi.org/10.1007/s10854-021-06249-y.

Address correspondence to E-mail: ahmadumar786@gmail.com 